ISSN 1991-8631

\title{
Caractérisation morphologique des populations de Sarotherodon melanotheron (Pisces, Cichlidae) en eaux douces et saumâtres au Sud Bénin
}

\author{
Siméon M. FAGNON ${ }^{1 *}$, Antoine CHIKOU ${ }^{2}$, Issaka YOUSSAO ${ }^{1}$ et Philippe LALEYE $^{2}$ \\ ${ }^{1}$ Université d'Abomey-Calavi, Ecole Polytechnique d'Abomey-Calavi, Département de Production et Santé \\ Animales. 01 BP 526 Cotonou, Bénin. \\ ${ }^{2}$ Université d'Abomey-Calavi, Faculté des Sciences Agronomiques, Laboratoire d'Hydrobiologie et \\ d'Aquaculture. 01 BP 526 Cotonou, Bénin. \\ *Auteur correspondant, E-mail : simeonfagnon@yahoo.fr, Tél : (00229) 97041228
}

\section{RESUME}

A partir de 900 spécimens de poissons, une étude de la caractérisation morphologique des populations de Sarotherodon melanotheron melanotheron a été réalisée dans six plans d'eau au sud et au centre du Bénin. Les données ont été collectées de juillet à septembre 2011 dans le Lac Nokoué, la Lagune de Porto Novo, la Lagune Côtière à Djègbadji, le Lac Ahémé, le Lac Toho et le barrage de la société sucrière (SUCOBE) à Savè. Le poids corporel, dix-sept (17) caractères métriques et onze (11) caractères méristiques relevés sur chaque individu ont permis d'analyser les variabilités morphologiques des populations des six (6) plans d'eau. Vingtsix (26) des 28 caractères morpho métriques étudiés se sont révélés discriminants. Parmi ceux-ci, les caractères métriques ont été les plus pertinents dans la discrimination de $S$. m. melanotheron et ont discriminé les populations de la Lagune de Porto-Novo, du Lac Ahémé et du barrage de SUCOBE entre elles, puis de celles du Lac Nokoué, du Lac Toho et de la Lagune Côtière à Djègbadji. L'étude constitue une base pour la détermination d'une souche performante de $S$. m. melanotheron à utiliser en pisciculture lagunaire au Bénin. (C) 2013 International Formulae Group. All rights reserved.

Mots clés: Sarotherodon melanotheron melanotheron, différenciation morphologique, eaux saumâtres, eaux douces, pisciculture lagunaire.

\section{INTRODUCTION}

Sarotherodon

melanotheron

melanotheron est une espèce euryhaline qui peut vivre et se reproduire dans une gamme de salinité très étendue de 0 à 90\%o (Albaret, 1987). Elle est donc adaptée aux estuaires, aux eaux saumâtres et aux eaux douces de l'Afrique de l'Ouest. Au Bénin, il s'agit des eaux saumâtres représentées par la Lagune de Porto-Novo, le Lac Nokoué, la Lagune Côtière à Djègbadji et le Lac Ahémé et des eaux douces représentées par le Lac Toho et le barrage de la société sucrière de Savè (SUCOBE). Elle présente en outre une forte tolérance aux faibles taux d'oxygène (Philippart et Ruwet, 1982) et un intérêt pour la pisciculture (Pauly, 1976; Sivalingam, 1976; Legendre, 1983; Legendre et Trebaol, 1996; Ouattara et al., 2005; Chikou et al., 2011). Malgré ses potentialités signalées, Oreochromis niloticus est la seule espèce du groupe tilapia élevée au Bénin. Or, cette 
espèce est strictement adaptée aux eaux douces continentales, donc confrontée aux problèmes liés à la tolérance de la salinité parfois élevée des lagunes. Il est important de valoriser nos milieux estuariens et lagunaires à travers la domestication d'espèces autochtones dont S. m. melanotheron. Cette domestication qui est en cours devra déboucher sur des souches performantes à mettre à la disposition des pisciculteurs lagunaires. Or, il semble que les souches à domestiquer ne sont toujours pas les mêmes et varient selon leur origine (eaux douces, eaux saumâtres). Ce travail vise principalement à identifier les différentes souches/populations de l'espèce à domestiquer pour la pisciculture lagunaire au Bénin.

\section{MATERIEL ET METHODES}

Cette étude a été conduite au Bénin de juillet à septembre 2011 dans six (6) plans d'eau dont deux (2) d'eau douce (Lac Toho et le barrage de SUCOBE) et quatre (4) d'eau saumâtre (la Lagune de Porto-Novo, le Lac Nokoué, la Lagune Côtière à Djègbadji, le Lac Ahémé) (Figure 1). L'échantillonnage a porté sur 900 individus de $S$. m. melanotheron issus des pêches artisanales. Par plan d'eau et par mois, 50 individus ont été collectés au hasard au niveau de chaque débarcadère. Aucune différenciation n'a été faite des différents engins utilisés pour capturer les poissons, ainsi que les différents sous habitats des plans d'eau au niveau desquels ceux-ci auraient été pêchés. Après l'échantillonnage, les poissons sont traités sur le terrain ou ramenés au laboratoire conservés dans la glace. Des mesures morpho métriques et pondérales sont ensuite réalisées sur chaque individu selon Paugy et al. (2004) et Adepo-Gourène et Gourène (2008).

\section{Caractères métriques}

Pour chaque spécimen, 17 mensurations sont relevées comme indiquées sur la Figure 2. Ce sont : longueur totale (LT), longueur standard (LS), hauteur du corps (HC), longueur de la tête (LTe), longueur du museau (LM), diamètre de l'œil (DO), largeur inter orbitaire (LIO), longueur pré dorsale (LPrD), longueur pré anale (LPrA), longueur pré pectorale (LPrP), longueur pré pelvienne (LPrPe), longueur de base de la nageoire dorsale (LD), longueur de base de la nageoire anale (LA), longueur de la nageoire pectorale (LNP), longueur de la nageoire pelvienne (LNPe), hauteur du pédoncule caudal (HPC), longueur dorso-anale (LDA).

\section{Caractères méristiques}

Onze (11) comptages sont effectués sur chaque spécimen. Il s'agit du : nombre de rayons mous dorsaux (RMD), nombre de rayons épineux dorsaux (RDD), nombre de rayons mous anaux (RMA), nombre de rayons épineux anaux (RDA), nombre d'écailles sur la ligne latérale supérieure (ELLS), nombre d'écailles sur la ligne latérale inférieure (ELLI), nombre d'écailles sur la ligne transversale (ELT), nombre d'écailles pré-dorsales (Epd), nombre d'écailles autour du pédoncule caudal (EPC), nombre d'écailles au niveau de l'opercule (EOp), nombre de branchiospines sur la partie inférieure du premier arc branchial (Bsp).

\section{Traitement des données}

Les données recueillies ont été traitées au moyen des logiciels suivants : Excel 2007, Statistica version 7.1 et Statview Version 5.1. Les données morphométriques ont été soumises à une Anova 1, analyse caractère par caractère, une Analyse Factorielle des Correspondances (AFC) et une Classification numérique. 


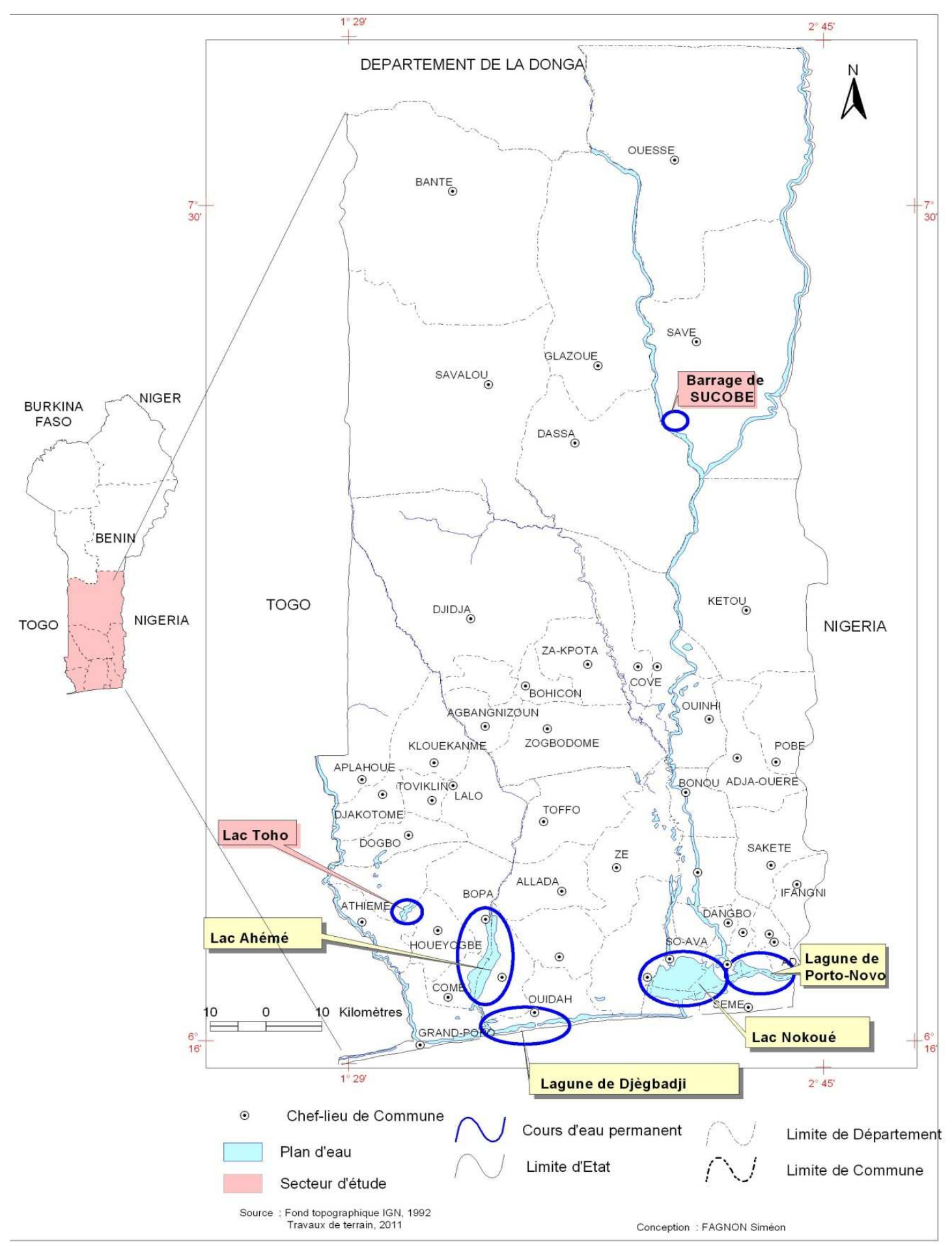

Figure 1: Situation géographique des milieux d'étude (Lagune de Porto-Novo, Lac Nokoué, Lagune Côtière à Djègbadji, Lac Ahémé, Lac Toho et barrage de SUCOBE). 


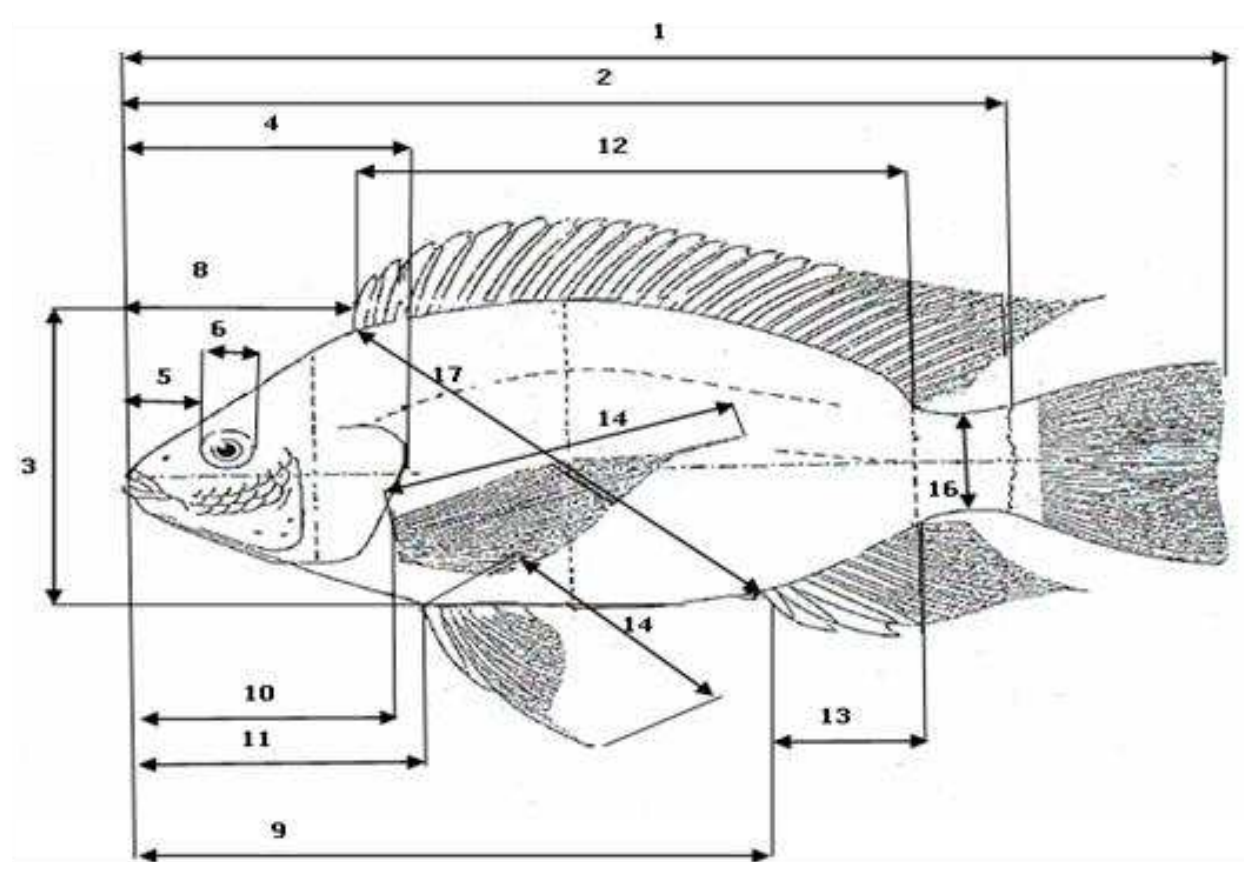

Figure 2: Mensurations effectuées sur chaque spécimen (les numéros d'ordre sur la figure représentent les caractères métriques correspondants) : 1 (LT), 2 (LS), 3 (HC), 4 (LTe), 5 (LM), 6 (DO), 7 (LIO), 8 (LPrD), 9 (LPrA), 10 (LPrP), 11 (LPrPe), 12 (LD), 13 (LA), 14 (LNP), 15 (LNPe), 16 (HPC), 17 (LDA).

\section{RESULTATS}

Pour évaluer les différences observées entre les 6 populations considérées, les 29 caractères ont été soumis à une Anova à un critère. A l'issue de cette analyse, 27 caractères ont permis d'observer une différence significative $(\mathrm{p}<0,05)$ entre ces populations.

Afin d'analyser les rapprochements possibles entre les 6 plans d'eau à partir des caractères morphométriques, une Analyse Factorielle des Correspondances (AFC) a été réalisée. Les données correspondent à une matrice brute composée des 6 plans d'eau et de 26 caractères ayant présenté une différence significative entre les stations. Ainsi, les deux caractères (RDA et RMA) ne présentant pas de différence significative entre les plans d'eau ont été exclus parce-que n'apportant pas d'information supplémentaire. Le poids étant une variable pondérale, il a été également exclu de cette analyse qui porte sur les variables morphométriques.
Le Tableau 1 présente les valeurs propres et les proportions d'informations concentrées sur les axes factoriels. Dans ce tableau, on peut noter la décroissance rapide des pourcentages d'inertie où le premier axe explique $92,94 \%$ des variations entre plan d'eau et le deuxième axe $4,33 \%$, ce qui donne au total pour les deux premiers axes un pourcentage d'inertie de $97,27 \%$. Ce qui est suffisant pour garantir une précision d'interprétation des relations entre caractères étudiés et plans d'eau.

La Figure 3 présente les résultats de l'AFC. Il nous permet de différencier 2 grands groupes de plans d'eau: le groupe constitué de la Lagune Côtière à Djègbadji, du Lac Nokoué et du Lac Toho et le groupe composé du Lac Ahémé, du barrage de SUCOBE et la Lagune de Porto-Novo. Le premier groupe cidessus est différencié par les caractères méristiques tels que : Eop, EPC, Epd, Bsp, ELT, ELLS, RMA, RDD, ELLI et le deuxième groupe se différencie par les 
caractères métriques : DO, LM, LIO, LA, HC, LNPe, Lte, LPrD, LPrP, LPrPe, LPrA, LT, LS, HPC, LDA.

A l'issue de ces résultats, les deux groupes ont été séparément considérés pour analyser en leur sein les caractères qui les réunissent. Ainsi, les deux groupes sont de nouveau soumis à une AFC: les caractères méristiques avec la Lagune de Djègbadji, du Lac Nokoué et du Lac Toho et les caractères métriques avec le barrage de SUCOBE, la Lagune de Porto-Novo et le Lac Ahémé.

\section{Discrimination au sein du groupe 1}

La Figure 4 illustre les résultats de l'AFC sur les variables métriques au sein du groupe 1. La matrice de données est constituée de 3 plans d'eau et de 17 caractères métriques.

Le Tableau 2 présente les valeurs propres et les proportions d'informations concentrées sur l'axe factoriel 1 et 2 de l'AFC. Dans ce tableau, on peut noter la décroissance rapide des pourcentages d'inertie où le premier axe explique 79,22\% des variations entre les 3 plans d'eau et le deuxième axe $20,77 \%$, ce qui donne au total pour les deux axes $100 \%$.

L'analyse de ce résultat de l'AFC sur les valeurs métriques permet de ressortir deux sous-groupes. Il s'agit du sous-groupe constitué du barrage de SUCOBE et de la Lagune de Porto-Novo et du sous-groupe constitué du Lac Ahémé.

Le dendrogramme appliqué sur les variables métriques nous a permis de confirmer ces 2 sous-groupes avec un coefficient de détermination de 53\%. Cette valeur de $\mathrm{R}^{2}$ retenue est suffisante pour obtenir des sous-groupes suffisamment distincts les uns des autres. Il s'agit du sousgroupe constitué du barrage de SUCOBE et de la Lagune de Porto-Novo et du sous- groupe constitué du Lac Ahémé. La Figure 5 illustre le dendrogramme de la première classe.

\section{Discrimination au sein du groupe 2}

La Figure 6 illustre les résultats de l'AFC sur les variables méristiques au sein du groupe 2. La matrice de données est constituée de 3 plans d'eau et de 9 caractères méristiques.

Le Tableau 3 présente les valeurs propres et les proportions d'informations concentrées sur les 2 axes factoriels de l'AFC des variables méristiques. Dans ce tableau, on peut noter la décroissance rapide des pourcentages d'inertie où le premier axe explique $75,97 \%$ des variations entre les 3 plans d'eau et le deuxième axe $24,03 \%$, ce qui donne au total pour les deux axes $100 \%$.

Le résultat issu de cette analyse nous permet de regrouper en deux sous-groupes ce grand groupe. Alors, on observe que la Lagune Côtière à Djègbadji est associée au Lac Nokoué tandis que le Lac Toho constitue à lui seul un sous-groupe.

Le dendrogramme appliqué sur les variables méristiques nous a permis de confirmer ces 2 sous-groupes avec un coefficient de détermination de 53\% (Figure 7). On y voit également 2 sous-groupes avec un coefficient de détermination suffisant pour obtenir des sous-groupes suffisamment distincts les uns des autres. Il s'agit du sousgroupe constitué du Lac Nokoué et de la Lagune Côtière à Djègbadji et du sous-groupe constitué du Lac Toho seul.

A l'issue de ces résultats, on constate que les deux dendrogrammes confirment les résultats issus de l'AFC et sépare les 6 populations en deux groupes au sein desquelles on distingue deux sous groupes. Le premier groupe est constitué de deux sous groupes dont la Lagune de Porto-Novo et le barrage de SUCOBE et le deuxième sous groupe est constituée du Lac Ahémé. Le deuxième groupe constitué aussi de deux sous groupes comporte le Lac Nokoué et la Lagune Côtière à Djègbadji comme un sous groupe et le Lac Toho comme le second sous groupe. 


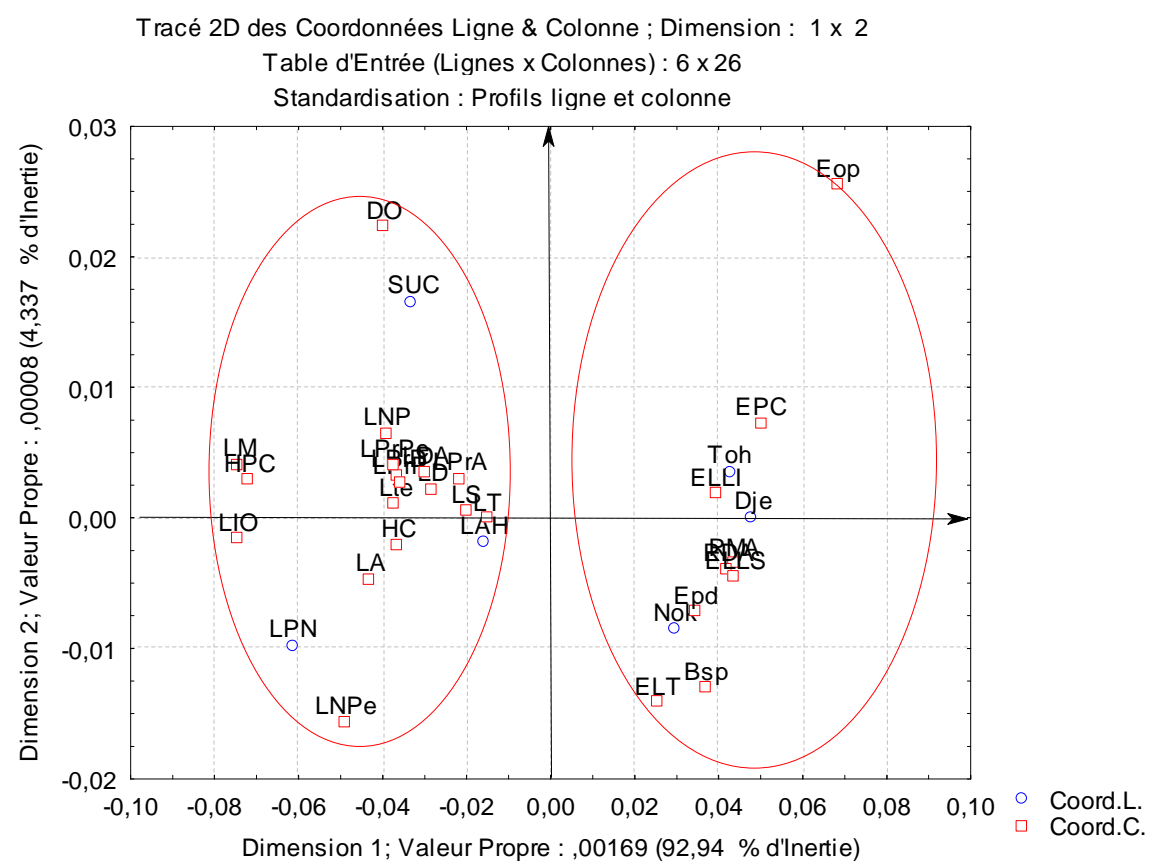

Figure 3: Résultats de l'Analyse Factorielle des Correspondances sur les variables morphométriques.

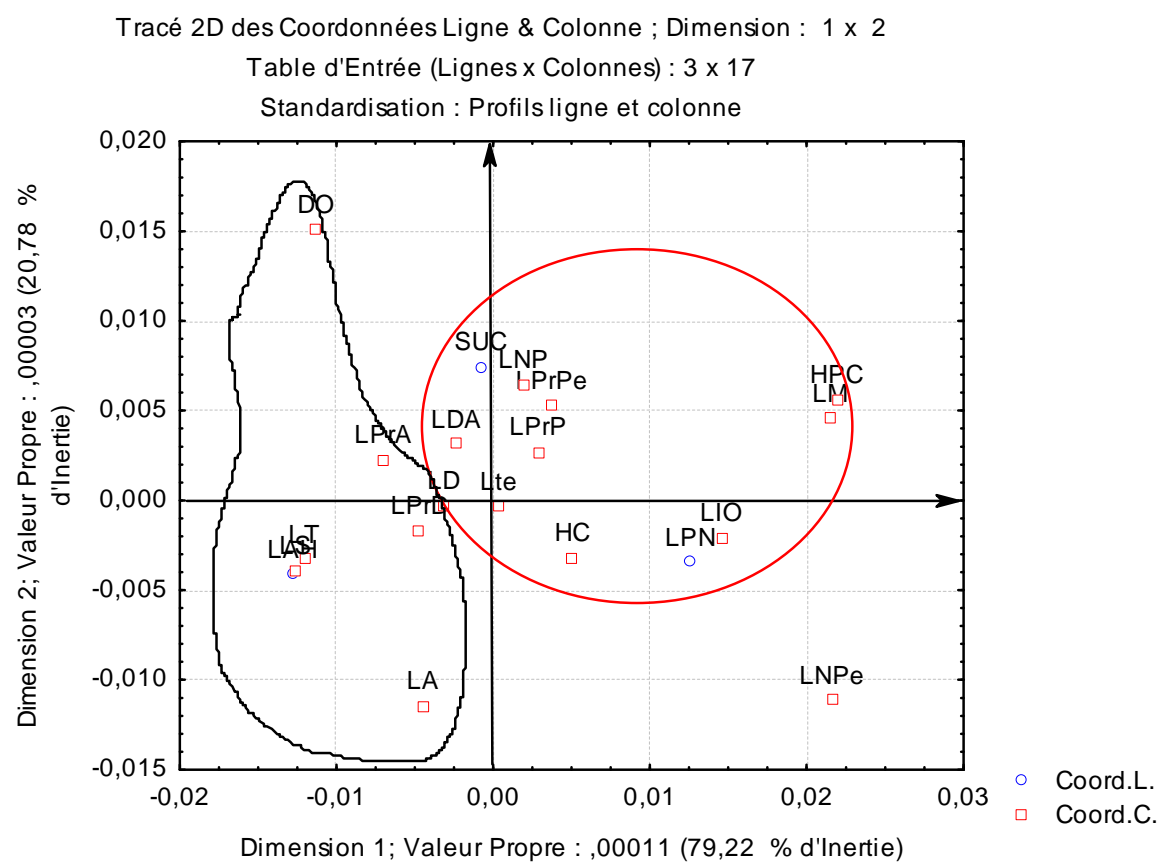

Figure 4: Résultats de l'Analyse Factorielle des Correspondances sur les variables métriques (classe de Porto-Novo, Ahémé et SUCOBE). 

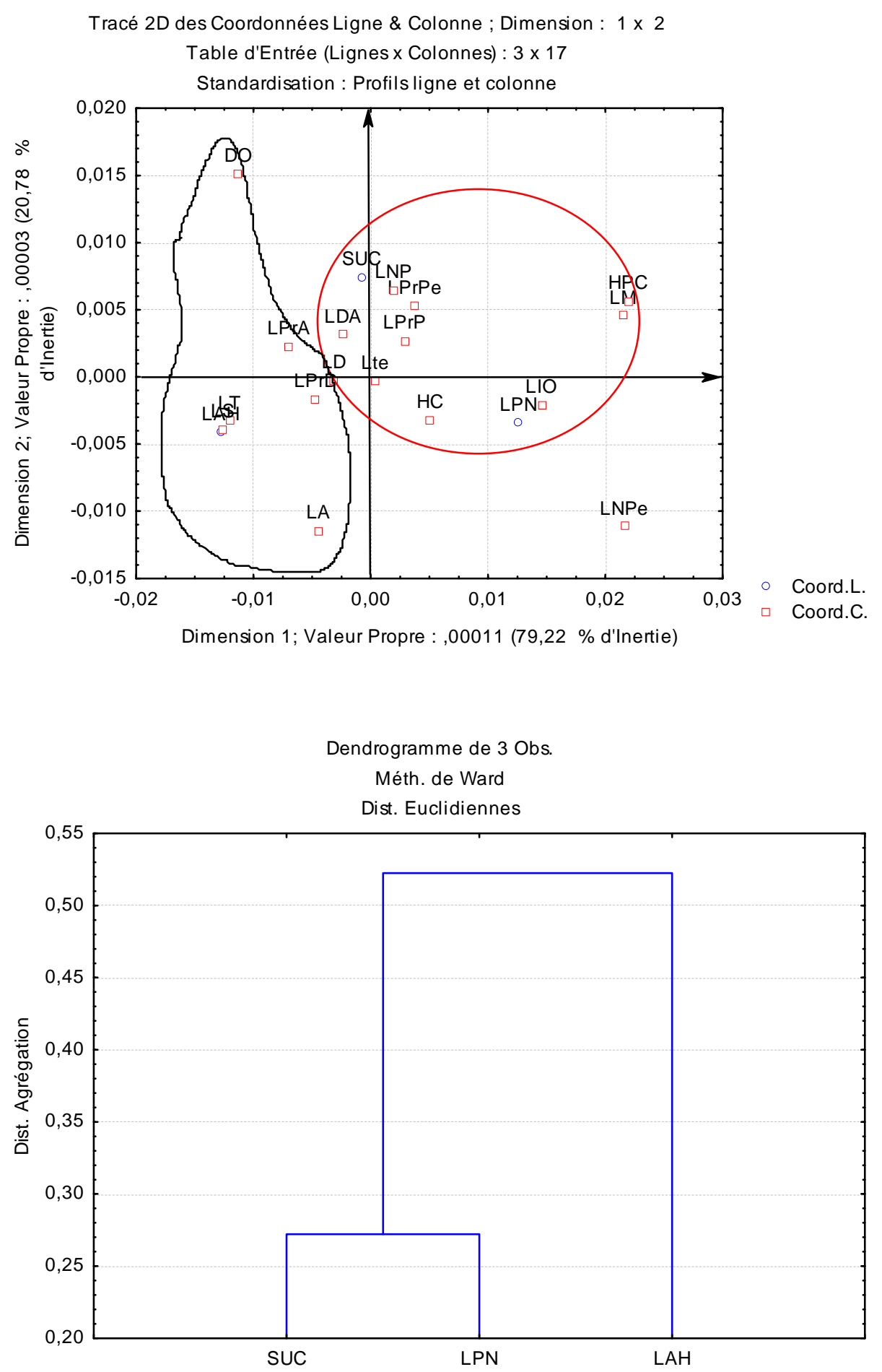

Figure 5: Dendrogramme relatif au regroupement des populations du $1^{\text {er }}$ groupe (Porto, SUCOBE et Ahémé). 


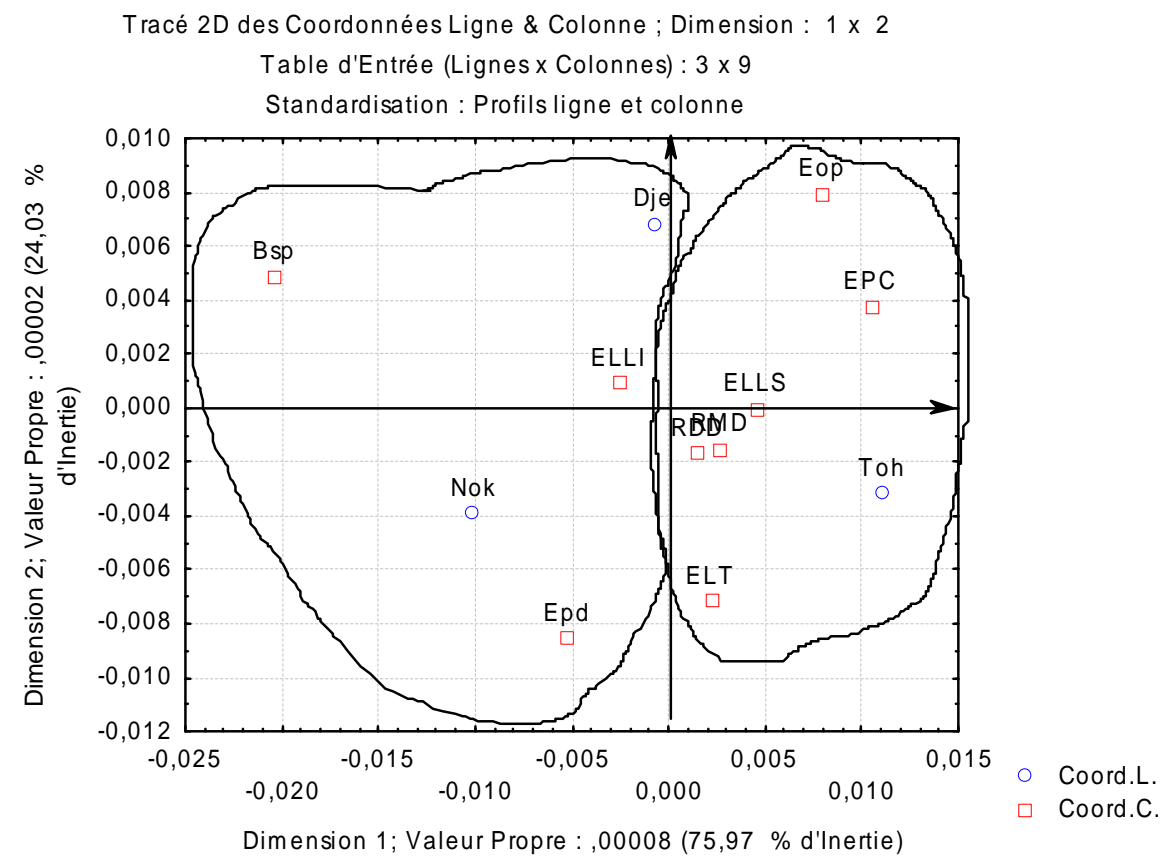

Figure 6: Résultats de l'Analyse Factorielle des Correspondances appliquées sur les variables méristiques.

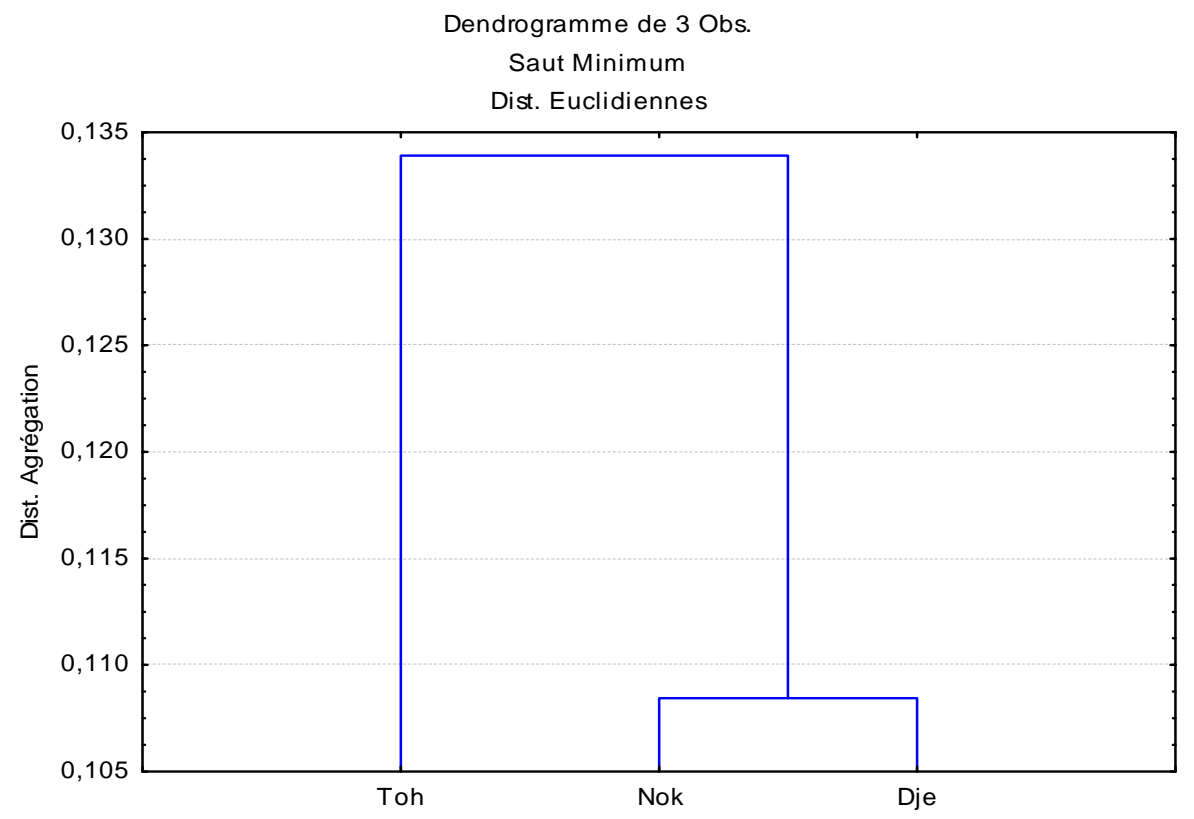

Figure 7: Dendrogramme relatif au regroupement des populations du deuxième groupe (Nokoué, Djègbadji et Toho). 
Tableau 1: Valeurs propres et proportions d'informations concentrées sur les axes de l'AFC.

\begin{tabular}{lccccc}
\hline Dim & Val sing & Val propre & \% inertie & \% cumulé & chi $^{2}$ \\
\hline 1 & 0,041140 & 0,001692 & 92,94048 & 92,9405 & 0,487337 \\
2 & 0,008887 & 0,000079 & 4,33708 & 97,2776 & 0,022742 \\
3 & 0,005485 & 0,000030 & 1,65199 & 98,9296 & 0,008662 \\
4 & 0,003819 & 0,000015 & 0,80107 & 99,7306 & 0,004200 \\
5 & 0,002215 & 0,000005 & 0,26938 & 100,0000 & 0,001413 \\
\hline
\end{tabular}

Tableau 2: Valeurs propres et proportions d'informations concentrées sur les 2 axes de l'AFC sur les variables métriques.

\begin{tabular}{lccccc}
\hline Dim & Val sing & Val propre & \% inertie & \% cumulé & chi $^{2}$ \\
\hline 1 & 0,010365 & 0,000107 & 79,22268 & 79,2227 & 0,009087 \\
2 & 0,005308 & 0,000028 & 20,77732 & 100,0000 & 0,002383 \\
\hline
\end{tabular}

Tableau 3: Valeurs propres et proportions d'informations concentrées sur les 2 axes de l'AFC sur les variables méristiques.

\begin{tabular}{lccccc}
\hline Dim & Val sing & Val propre & \% inertie & \% cumulé & chi $^{2}$ \\
\hline 1 & 0,008694 & 0,000076 & 75,96948 & 75,9695 & 0,005285 \\
2 & 0,004890 & 0,000024 & 24,03052 & 100,0000 & 0,001672 \\
\hline
\end{tabular}

\section{DISCUSSION}

De nombreuses études ont signalé la présence de la sous espèce $S$. melanotheron melanotheron dans les plans d'eau du Bénin (Pouyaud et Agnèse, 1994; AhouansouMontcho, 2003 ; Lalèyè et al., 2003; Lalèyè et al., 2004 ; Paugy et al., 2004; Villanueva, 2004; Yacoubou, 2005; Okpeicha, 2010). Selon Trewavas (1983), plusieurs caractères méristiques permettent de caractériser cette sous espèce. Il s'agit du : nombre de rayons à l'anale, du nombre de rayons à la dorsale, du nombre de branchiospines sur la partie inférieure de l'arc branchial, etc. Les populations de $S$. m. melanotheron que nous avons étudiées s'étendent en nombre de rayons mous dorsaux de 10 à 12 et en nombre de rayons durs dorsaux de 15 à 17 . Ses valeurs obtenus sont conformes à celles de Trewavas (1983) qui situe le nombre de rayons dorsaux entre 25 et 29 avec une étendue de 10-12 pour les rayons mous et de 15-17 pour les rayons durs. Quant au nombre de rayons anaux, il les situe entre 10 et 13 avec le nombre de rayons mous variant entre 7 et 10 et le nombre de rayons durs égal à 3. Nos échantillons répondent bien à sa limite par rapport au nombre de rayons durs anaux sauf un léger écart de 7 à 10 obtenu au niveau du nombre de rayons mous anaux. Le nombre de 
branchiospines sur le premier arc branchial s'étend de 13 à 19 conformément à la description de Trewavas (1983). La conformité de ces différents caractères traduit en partie l'appartenance effective de nos échantillons à la sous espèce $S . m$. melanotheron.

Des 28 caractères morphométriques analysés, 26 permettent de différencier significativement les six populations étudiées. Les caractères métriques tels que: LT, LS, HC, Lte, LM, DO, LIO, LPrD, LPrA, LPrP, LPrPe, LD, LA, LNP, LNPe, HPC et LDA permettent de discriminer les populations du barrage de SUCOBE, de la Lagune de PortoNovo et du Lac Ahémé entre elles d'une part et de les différencier du deuxième groupe qui est constituée du Lac Nokoué, du Lac Toho et de la Lagune Côtière à Djègbadji d'autre part. Par contre, ce deuxième groupe est discriminé en son sein et opposé au premier groupe par les caractères méristiques. Il s'agit de Eop, EPC, Epd, Bsp, ELT, ELLS, RMA, RDD et ELLI.

L'existence d'une différenciation morphologique entre les 6 populations étudiées a été clairement mise en évidence par les différentes analyses (Anova, AFC et classification numérique). Tous les caractères métriques ont contribué à cette séparation. Par contre d'autres se sont avérés sans influence au niveau des caractères méristiques. Les travaux d'Adepo-Gourène et Gourène (2008) ont déjà relevé les variables métriques comme pertinentes dans la discrimination de $S . m$. melanotheron en l'occurrence la longueur de la tête pour $O$. niloticus (Gourene et Teugels, 1993) et le diamètre de l'œil pour les tilapias en général (Huber et al., 1997; Schliewen et al., 2001). Ces descripteurs identifiés pertinents ont discriminé les populations de la Lagune de Porto-Novo, du Lac Ahémé et du barrage de SUCOBE entre elles puis des trois autres populations. Ce qui confirme leur considération comme un trait écologique clé lié à l'habitat des poissons (Adepo-Gourène et Gourène, 2008). Selon Barlow (1961) et Dyne et al. (1999), la variation de certains des descripteurs métriques (hauteur du corps, hauteur du pédoncule caudal, longueur du museau, longueur de la nageoire pectorale) et méristiques serait phénotypique car liée à l'environnement. L'influence de ces caractères dans la discrimination des 6 populations étudiées traduit le rapprochement de facteurs écologiques ou environnementaux de certains plans d'eau.

De façon générale, l'influence de la salinité a été moindre dans la discrimination des 6 populations étudiées. Cela pourrait être la conséquence de la dominance d'autres facteurs environnementaux sur ce paramètre.

Toutefois, certains plans d'eau sont interconnectés par les eaux marines et les eaux fluviales. Cette connexion a-t-elle un effet sur les différents regroupements observés dans cette étude ? Ces éléments constituent de nouvelles orientations de recherches à découvrir pour apprécier l'influence de l'environnement écologique sur la sousespèce étudiée.

\section{REMERCIEMENTS}

Les auteurs remercient l'équipe du Laboratoire d'Hydrobiologie et d'Aquaculture de la Faculté des Sciences Agronomiques, les pêcheurs et les mareyeuses qui ont contribué à la constitution et au traitement des échantillons des différents plans d'eau.

\section{REFERENCES}

Adepo-Gourène B, Gourène G. 2008. Différenciation morphologique des populations naturelles d'une sous espèce de tilapia Sarotherodon melanotheron melanotheron Rüppell, 1852 (Teleostei ; Cichlidae) de Côte d'Ivoire. Sciences et nature, 5(1) : 15-27.

Ahouansou-Montcho S. 2003. Etude de l'écologie et de la production halieutique 
du lac Toho au Bénin. Mémoire de DESS/FSA, Université d'Abomey-Calavi, Bénin, p. 87.

Albaret JJ. 1987. Les peuplements de poissons de la Casamance (Sénégal) en période de sécheresse. Rev. Hydrobiol. Trop., 20: 291310.

Barlow GW. 1961. Causes and significance of morphological variation in fishes. Systematic Zoology., 10: 105-117.

Chikou A. 2011. Effet du ratio «mâles : femelles » sur la production d'alevins par des géniteurs de Sarotherodon melanotheron élevés en bassins. Communication scientifique présentée au $3^{\text {e }}$ colloque de l'Université d'AbomeyCalavi des Sciences, Cultures et Technologies. Programme des Résumés, 292-293.

Dyne J, Magnan P, Bernatchez L, Rodriguez MA. 1999. Genetic and morphological variation between two forms of lacustrine brook charr. J. Fish Biol., 54, 955-972.

Gourène G, Teugels GG. 1993. Différenciation morphologique de souches des tilapias: Oreochromis niloticus et $O$. aureus (Teleostei ; Cichlidae) utilisées en pisciculture. Cybium., 17(4): 343-355.

Huber R, Van Staden M, Kaufman LS, Liem KF. 1997. Microhabitat use, trophic patterns, and, the evolution of brain structure in African cichlids. Br. Behav. Evol., 50: 167-182.

Lalèyè $\mathrm{P}$, Niyonkuru $\mathrm{C}$, Moreau $\mathrm{J}$, Teugels GG. 2003. Spatial and seasonal Distribution of the ichthyofauna of Lake Nokoué, Benin, West Africa. African Journal of Aquatic Sciences, 28(2): 151161.

Lalèyè $\mathrm{P}$, Chikou $\mathrm{A}$, Philippart JC, Teugels GG, Vandewalle P. 2004. - Etude de la diversité ichtyologique du bassin du fleuve Ouémé au Bénin (Afrique de l'Ouest). Cybium., 28(4): 329-339.
Legendre M. 1983. Observations préliminaires sur la croissance et le comportement en élevage de Sarotherodon melanotheron (Rüppel, 1852) et de Tilapia guineensis (Bleeker, 1862) en lagune Ebrié (Côte d'Ivoire). Doc. Sci. Cent. Rech. Océanogr. Abidjan ORSTOM, 14: 1-36.

Legendre M, Trebaol L. 1996. Efficacité de l'incubation buccale et fréquence de ponte de Sarotherodon melanotheron (Rüppel, 185l) en milieu d'élevage (lagune Ebrié, Côte d'Ivoire). Pullin RSV, Lazard J, Legendre M, Amon Kotias JB, Pauly D (eds). Le troisième symposium international sur le tilapia en aquaculture. ICLARM conf. Proc., 41, - 630 p.

Okpeïcha S. 2010. Biodiversité et exploitation des poissons du barrage de SUCOBE dans la commune de Savè au Bénin. Mémoire de Master en Hydrobiologie Appliquée à la Faculté des Sciences et Techniques de l'Université d'Abomey-Calavi, p. 43.

Ouattara NI, N'Douba V, Kone T, Snoeks J, Philippart JC. 2005. Performances de croissance d'une souche isolée du Tilapia estuarien Sarotherodon melanotheron (perciformes, Cichlidae) en bassins en beton, en etangs en terre et en Cages flottantes. Ann. Univ. M. Ngouabi., 6(1): 113-119.

Paugy D, Levêque C, Teugels G. 2004. Poissons d'Eaux Douces et Saumâtres de l'Afrique de l'Ouest. Faune Tropicale. IRD: Paris; $815 \mathrm{p}$.

Pauly D. 1976. The biology, fishery and potential for aquaculture of Tilapia melanotheron in a small West African lagoon. Aquaculture, 7: 33-49.

Philippart J, Ruwet JC. 1982. Ecology and distribution of Tilapias. In The Biology and Culture of Tilapias, Pullin RSV, Lowemcconnell RH (eds). ICLARM Conf. Proc.: Manilla, Philippines; 16-60.

Pouyaud L, Agnèse JF. 1994. Différenciation génétique des populations de Sarotherodon 
melanotheron, Rüppell, 1853. Atelier Biodiversité et Aquaculture, p. 8.

Schilewen U, Rassmann K, Markmann M, Markert J, Kocher T, Tautz D. 2001. Genetic, and ecological divergence of a monophyletic cichlid species pair under fully sympatric conditions in Lake Ejagham, Cameroon. Mol. Ecol., 10: 1471-1488.

Sivalingam S. 1976. The biology of cultivable brackish water and marine finfish in Africa, p. 283-291.In Proceedings of the FAO/C1FA Symposium on Aquaculture in Africa, Accra, Ghana. CIFA Tech, p. 4.

Trewavas E. 1983. Tilapiine Fishes of the Genera Sarotherodon, Oreochromis, and
Danakilia. British Museum (Natural History): London; 583.

Villanueva MC. 2004. Biodiversité et relation trophiques dans quelques milieux estuariens et lagunaires de l'Afrique de l'Ouest: Adaptation aux pressions environnementales. Thèse de doctorat de l'Ecole Nationale Supérieure Agronomique de Toulouse, France, p. 246. Yacoubou R. 2005. Contribution à l'évaluation écologique des zones humides du site RAMSAR 1080 au Sud-Bénin : cas de la lagune de Porto-Novo. Thèse d'Ingénieur agronome à l'Université d'Abomey-Calavi, Bénin, p. 104. 